\title{
Comparative Numerical Analysis of Magnetic and Optical Radiation Propagation in Adult Human Head
}

\author{
Noé Ortega-Quijano, Félix Fanjul-Vélez, Irene Salas-García, José Luis Arce-Diego \\ Applied Optical Techniques Group, Electronics Technology, Systems and Automation Engineering \\ Department, University of Cantabria, Avenida de los Castros S/N, 39005 Santander, Spain \\ ortegan@unican.es; arcedj@unican.es
}

\begin{abstract}
In this work, magnetic and optical propagation in human head are modeled by FDTD and Monte Carlo methods. Both of them use a realistic high-resolution three-dimensional human head mesh. The numerical methods are applied to the analysis of magnetic and optical radiation distribution in the brain using different sources. The results show the characteristics of both types of stimulation, and highlight the spatial selectivity achieved by optical sources, which entails a high potential for illuminating specific brain regions. The presented approach can be applied for predictive purposes in magnetic stimulation techniques and in the emerging field of optical brain stimulation.
\end{abstract}

Keywords: Predictive modeling, FDTD, Magnetic Transcranial Brain Stimulation, Monte Carlo, optical brain stimulation.

\section{INTRODUCTION}

Population ageing is a global trend in developed countries. It is estimated that the number of people older than 65 years will rise by $70 \%$ by 2050 in the EU [1]. Neurodegenerative diseases are strongly related to ageing, and constitute a major cause of dementia and personal mobility loss. There are multiple causes and factors that can provoke neurodegenerative diseases, but most of them entail neuronal synapses weakening and neurotransmitters malfunctions. It is well-known that nervous tissue stimulation can be achieved by several type of energy sources, which lead to electrical, magnetical, chemical, thermical, mechanical and optical stimulation methods [2]. The positive effects generated by stimulation processes in nervous tissue enables to use it as a therapeutic method.

This work in focused on stimulation by two different types of electromagnetic sources, namely magnetic and optical sources. Among the magnetic stimulation techniques, Transcranial Brain Stimulation (TBS) is one of the most widely used ones [3]. Its positive effects on Parkinson disease have been demonstrated, and further research on its application to Alzheimer disease is currently under development [4,5]. Regarding optical stimulation, Infrared Neural Stimulation (INS) is a novel technique which is achieving very promising results, and thus has a high potential for its clinical use $[2,6]$.

We present the application of two different numerical methods for modeling electromagnetic propagation in a realistic model of adult human head. A specific Finite Difference Time Domain (FDTD) method is used for modeling lowfrequency magnetic propagation, while the Monte Carlo method is applied to solve optical propagation with different optical sources. We show several results that enable to observe the main characteristics of each type of stimulation. The analysis performed in this work constitute the first step towards the development of comprehensive predictive models that could enable to determine the magnetic or optical radiation distribution in the brain, in order to appropriately control radiation parameters for enhancing and optimizing the stimulation process.

In Section 2, the well-known FDTD method is briefly described. Section 3 summarizes the main characteristics and steps of the Monte Carlo method. Subsequently, in Section 4 these numerical methods are particularized for modeling magnetic and optical propagation in human brain. Results for several types of sources are given. The characteristics of both techniques are comparatively presented. Finally, the main conclusions are presented in Section 5.

Medical Laser Applications and Laser-Tissue Interactions VI, edited by Lothar D. Lilge, Ronald Sroka,

Proc. of OSA Biomedical Optics-SPIE Vol. 8803, 880308 • (c) 2013 OSA-SPIE

CCC code: $1605-7422 / 13 / \$ 18 \cdot$ doi: $10.1117 / 12.2033544$ 


\section{FDTD METHOD}

One of the most powerful numerical methods for studying electromagnetic propagation through different types of media is the finite-difference time-domain (FDTD) method. FDTD is widely used for numerically solving Maxwell's equations. Although it entails a high computing time, its high accuracy and its versatility makes it an essential method for solving electromagnetic propagation in many applications. In particular, this computational method has demonstrated to be a robust and efficient method for the calculation of magnetic propagation in biological tissues [7,8].

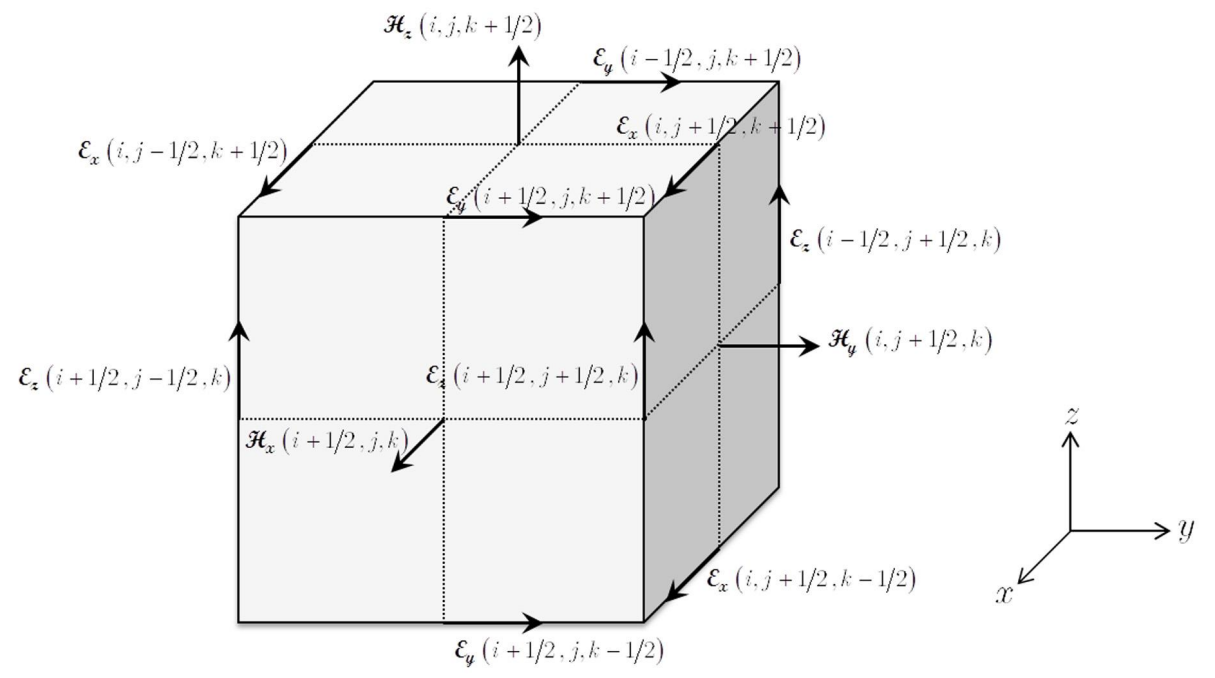

Fig. 1. Yee lattice and electromagnetic field vectors involved in the FDTD method.

FDTD method constitutes a direct implementation of Maxwell's equations in the time domain. Firstly, a spatial discretization is performed using a rectangular mesh of $\left(N_{x}, N_{y}, N_{z}\right)$ cubes with edge lengths of $\Delta_{i j k}$. Generic stability conditions impose a minimum number of 20 cells for a wavelength. Fig.1 represents the Yee lattice (i.e. the basic element of the spatial mesh), in which the electromagnetic field vectors involved in the FDTD method have been depicted. Additionally, time is also discretized with a step of $\Delta t$. Stability conditions impose a maximum time step of:

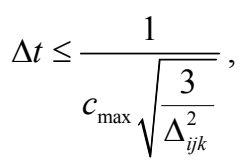

where $c_{\max }$ is the electromagnetic wave propagation speed in the medium. From Maxwell's equations, and adapting the partial derivatives to the spatial and temporal discretization mentioned above, the equations for calculating the electric and magnetic fields for each position and time instant are obtained [7]. In particular, the magnetic field components (defined in the cube face centers) are obtained for the intervals between two consecutive time instants (denoted by the superscript) by the following equations:

$$
\begin{aligned}
& \left.H_{x}\right|_{i, j, k} ^{n+1 / 2}=\left.\left.H_{1}^{x}\right|_{i, j, k} H_{x}\right|_{i, j, k} ^{n-1 / 2}+\left.H_{2}^{x}\right|_{i, j, k}\left(\left.E_{y}\right|_{i, j, k+1} ^{n}-\left.E_{y}\right|_{i, j, k} ^{n}+\left.E_{z}\right|_{i, j, k} ^{n}-\left.E_{z}\right|_{i, j+1, k} ^{n}\right), \\
& \left.H_{y}\right|_{i, j, k} ^{n+1 / 2}=\left.\left.H_{1}^{y}\right|_{i, j, k} H_{y}\right|_{i, j, k} ^{n-1 / 2}+\left.H_{2}^{y}\right|_{i, j, k}\left(\left.E_{z}\right|_{i+1, j, k} ^{n}-\left.E_{z}\right|_{i, j, k} ^{n}+\left.E_{x}\right|_{i, j, k} ^{n}-\left.E_{x}\right|_{i, j, k+1} ^{n}\right), \\
& \left.H_{z}\right|_{i, j, k} ^{n+1 / 2}=\left.\left.H_{1}^{z}\right|_{i, j, k} H_{z}\right|_{i, j, k} ^{n-1 / 2}+\left.H_{2}^{z}\right|_{i, j, k}\left(\left.E_{x}\right|_{i, j+1, k} ^{n}-\left.E_{x}\right|_{i, j, k} ^{n}+\left.E_{y}\right|_{i, j, k} ^{n}-\left.E_{y}\right|_{i+1, j, k} ^{n}\right),
\end{aligned}
$$

where coefficients $H_{1}^{x}$ and $H_{2}^{x}$ are given by: 


$$
\begin{aligned}
& \left.H_{1}^{x}\right|_{i, j, k}=\frac{1-\rho_{i, j, k}^{x} \Delta t\left(2 \mu_{i, j, k}^{x}\right)^{-1}}{1+\rho_{i, j, k}^{x} \Delta t\left(2 \mu_{i, j, k}^{x}\right)^{-1}}, \\
& \left.H_{2}^{x}\right|_{i, j, k}=\frac{\Delta t\left(\Delta_{i j k} \mu_{i, j, k}^{x}\right)^{-1}}{1+\rho_{i, j, k}^{x} \Delta t\left(2 \mu_{i, j, k}^{x}\right)^{-1}} .
\end{aligned}
$$

Coefficients $H_{1}^{y}, H_{2}^{y}, H_{1}^{z}$ and $H_{2}^{z}$ are analogously defined. Magnetic permeability $\mu$ and magnetic losses $\rho$ are defined in the cube nodes $(i, j, k)$. Regarding the electric field (defined in the edges centers), their components for each time instant are:

$$
\begin{aligned}
& \left.E_{x}\right|_{i, j, k} ^{n+1}=\left.\left.E_{1}^{x}\right|_{i, j, k} E_{x}\right|_{i, j, k} ^{n}+\left.E_{2}^{x}\right|_{i, j, k}\left(\left.H_{z}\right|_{i, j, k} ^{n+1 / 2}-\left.H_{z}\right|_{i, j-1, k} ^{n+1 / 2}+\left.H_{y}\right|_{i, j, k-1} ^{n+1 / 2}-\left.H_{y}\right|_{i, j, k} ^{n+1 / 2}\right), \\
& \left.E_{y}\right|_{i, j, k} ^{n+1}=\left.\left.E_{1}^{y}\right|_{i, j, k} E_{y}\right|_{i, j, k} ^{n}+\left.E_{2}^{y}\right|_{i, j, k}\left(\left.H_{x}\right|_{i, j, k} ^{n+1 / 2}-\left.H_{x}\right|_{i, j, k-1} ^{n+1 / 2}+\left.H_{z}\right|_{i-1, j, k} ^{n+1 / 2}-\left.H_{z}\right|_{i, j, k} ^{n+1 / 2}\right), \\
& \left.E_{z}\right|_{i, j, k} ^{n+1}=\left.\left.E_{1}^{z}\right|_{i, j, k} E_{z}\right|_{i, j, k} ^{n}+\left.E_{2}^{z}\right|_{i, j, k}\left(\left.H_{y}\right|_{i, j, k} ^{n+1 / 2}-\left.H_{y}\right|_{i-1, j, k} ^{n+1 / 2}+\left.H_{x}\right|_{i, j-1, k} ^{n+1 / 2}-\left.H_{x}\right|_{i, j, k} ^{n+1 / 2}\right) .
\end{aligned}
$$

In the previous expressions, coefficients $E_{1}^{x}$ y $E_{2}^{x}$ are given by:

$$
\begin{aligned}
& \left.E_{1}^{x}\right|_{i, j, k}=\frac{1-\sigma_{i, j, k}^{x} \Delta t\left(2 \varepsilon_{i, j, k}^{x}\right)^{-1}}{1+\sigma_{i, j, k}^{x} \Delta t\left(2 \varepsilon_{i, j, k}^{x}\right)^{-1}}, \\
& \left.E_{2}^{x}\right|_{i, j, k}=\frac{\Delta t\left(\Delta_{i j k} \varepsilon_{i, j, k}^{x}\right)^{-1}}{1+\sigma_{i, j, k}^{x} \Delta t\left(2 \varepsilon_{i, j, k}^{x}\right)^{-1}} .
\end{aligned}
$$

Dielectric permittivity $\varepsilon$ and conductivity $\sigma$ are defined in the center of each cube. Coefficients for the remaining spatial components are defined in a similar way. For inhomogeneous media, it is necessary to calculate the effective properties of the simple, in order to ensure the continuity of the tangent field components. Therefore, the effective permittivity and permeability for the calculation of the field components along the $x$ direction can be calculating by:

$$
\begin{aligned}
\mu_{i, j, k}^{x} & =\frac{\mu_{i, j, k}+\mu_{i, j+1, k}+\mu_{i, j, k+1}+\mu_{i, j+1, k+1}}{4}, \\
\varepsilon_{i, j, k}^{x} & =\frac{\varepsilon_{i, j, k}+\varepsilon_{i, j-1, k}+\varepsilon_{i, j, k-1}+\varepsilon_{i, j-1, k-1}}{4} .
\end{aligned}
$$

The equations for the remaining directions, as well as those for the magnetic losses and conductivity, can be analogously obtained. Regarding the source, a great number of electromagnetic sources can be modeled. In the FDTD method used in this work, we have used a simple magnetic source: an air core magnetic coil that models an elemental magnetic dipole. This source can be arbitrarily oriented, and its implementation is performed by the double-closed current loop model described in [9]. Finally, the boundary conditions have been implemented by a combination of Mur's Absorbing Boundary Conditions and Superabsorption Conditions [7], which avoid reflections and calculation errors in the edges of the modeled volume.

\section{MONTE CARLO METHOD}

Monte Carlo method is a widely used approach for modeling light propagation in random media, among which biological tissues are a remarkable example $[10,11]$. It constitutes a stochastic method for statistically solving photon propagation in samples where scattering dominates over absorption (i.e. turbid media). The method relies on the 
determination of several probability distributions based on a comprehensive model of light propagation in turbid media [10]. Due to the statistical nature of the method, a large number of photons is needed in order to ensure the accuracy of the results. The flow chart of the Monte Carlo method is included in Fig. 1.

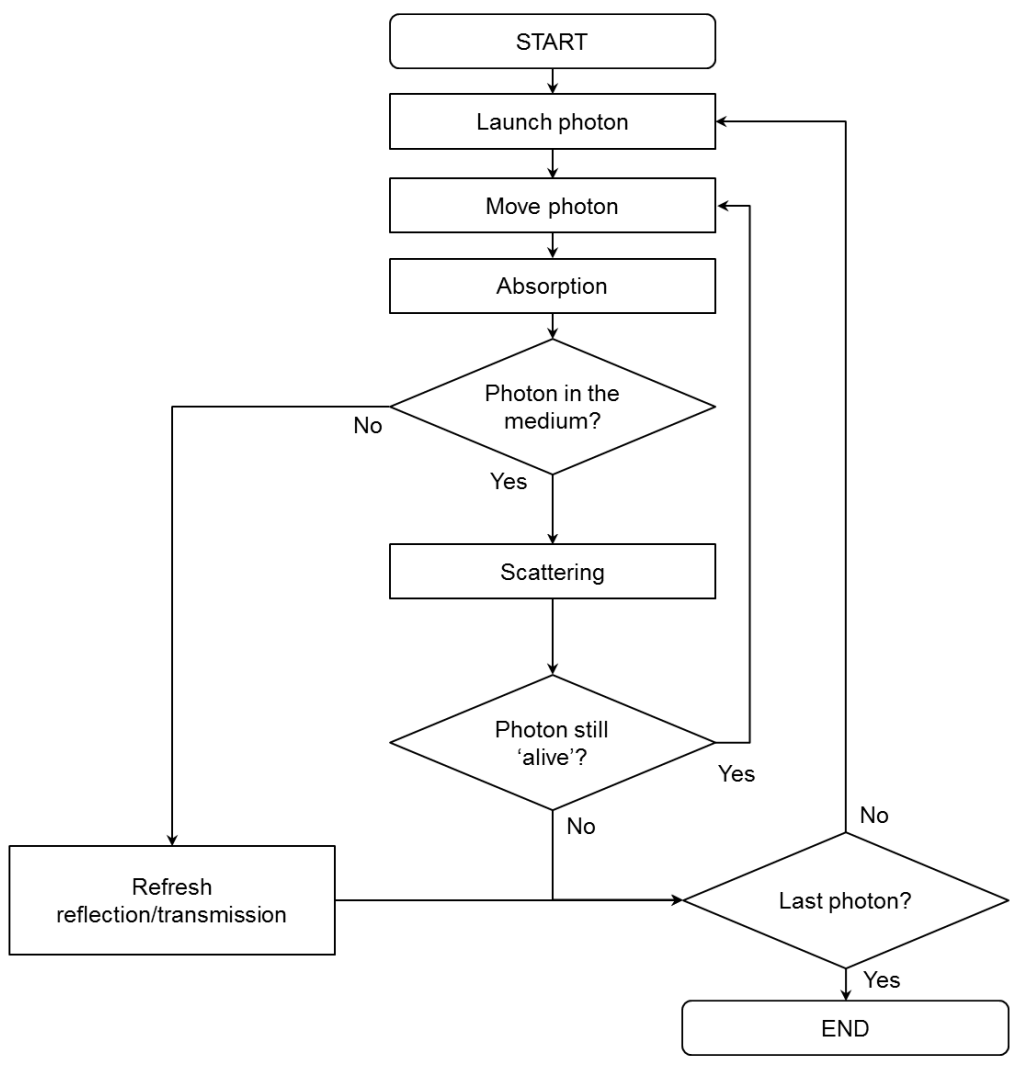

Fig. 2. Monte Carlo method flow chart.

The first step of the method is launching a packet of photons, which is initially given a weight $W=1$, a position $\vec{r}$, and a propagation direction $\hat{n}=\left(\begin{array}{lll}n_{x} & n_{y} & n_{z}\end{array}\right)$. Then, the photon displacement $\Delta s$ is calculated for each step by:

$$
\Delta s=\frac{-\ln \left(\xi_{1}\right)}{\mu_{e}},
$$

where $\mu_{e}$ is the extinction coefficient of the medium [12], and $\xi_{1} \in(0,1]$ is a pseudo-random number. The new position of the photon packet is obtained directly from the displacement and the unitary propagation vector:

$$
\vec{r}^{\prime}=\vec{r}+\Delta s \cdot \hat{n} .
$$

The effect of absorption in each displacement step is modeled by the following reduction in the packet weight:

$$
\Delta W=\frac{\mu_{a}}{\mu_{e}} W .
$$

The statistical sampling implemented in the displacement calculation implies that, if the photon continues in the medium, a scattering event takes place. The essential aspect in this step is to calculate the polar angle $\theta$ and the azimuthal angle $\phi$ of the scattered photon packet, that modify its propagation direction as a result of the elastic electromagnetic interaction of the photon packet with the scattering particle. The probability distribution function can be calculated by 
means of the Mie theory. However, in the Monte Carlo method it is usually modeled by the Henyey-Greenstein phase function:

$$
p(\cos \theta)=\frac{1-g^{2}}{2\left(1+g^{2}-2 \cos \theta\right)^{3 / 2}},
$$

where $g$ is the scattering anisotropy [12]. Therefore, the polar scattering angle is given by:

$$
\theta= \begin{cases}\operatorname{acos}\left\{\frac{1}{2 g}\left[1+g^{2}-\left(\frac{1-g^{2}}{1-g+2 g \xi_{2}}\right)^{2}\right]\right\}, & g \neq 0, \\ \operatorname{acos}\left(2 \xi_{2}-1\right) & , g=0\end{cases}
$$

In these equations, $\xi_{2}$ is a pseudo-random number with the same characteristics as $\xi_{1}$. Additionally, it is assumed that the azimuthal angle has a uniform probability distribution:

$$
\phi=2 \pi \xi_{3} .
$$

According to both angles, the new direction vector of the photon packet is refreshed using the following equations:

$$
\begin{gathered}
n_{x}^{\prime}=n_{x} \cos \theta+\frac{\left(n_{x} n_{z} \cos \phi-n_{y} \sin \phi\right)}{\left(1-n_{z}^{2}\right)} \sin \theta, \\
n_{y}^{\prime}=n_{y} \cos \theta+\frac{\left(n_{y} n_{z} \cos \phi+n_{x} \sin \phi\right)}{\left(1-n_{z}^{2}\right)} \sin \theta, \\
n_{z}^{\prime}=n_{z} \cos \theta-\left(1-n_{z}^{2}\right) \cos \phi \sin \theta .
\end{gathered}
$$

These steps are repeated till the photon packet is absorbed (according to the minimum weight threshold fixed a priori) or transmitted/backscattered. The process is performed for a high number of photon packets (typically around $10^{6}$ ). It should be noted that the presence of anisotropy or multiple layers require additional equations for modeling such effects [10].

\section{ELECTROMAGNETIC PROPAGATION MODELING IN A REALISTIC MODEL OF ADULT HUMAN HEAD}

The FDTD method has been widely used during the last decade for modeling magnetic propagation in human brain [13], especially for dosimetric applications. The FDTD method directly solves Maxwell's equations in the time domain. As a consequence, it is valid for arbitrary electromagnetic radiation. However, its application for the study of magnetic propagation in human head for brain stimulation at low imposes some difficulties. The fact that the frequencies commonly used in TBS are very low (roughly between $0.1 \mathrm{~Hz}$ and $10 \mathrm{KHz}$, although the range is commonly restricted to 0.1-100 Hz) makes it computationally unfeasible to perform a direct implementation of the FDTD method. In such situations, the problem can be solved by the frequency scaling method [13]. This method takes advantage of the quasistatic nature of the modeled situation. In particular, it is valid when the modeled volume is at least 10 times lower than the wavelength, and $|\sigma+i \omega \varepsilon|>>\omega \varepsilon_{0}$ [13]. Both conditions are verified for the case of brain tissue. According to such approach, the FDTD can be performed at a frequency $f^{\prime}$ higher than the frequency of interest $f$, and subsequently perform the following scaling operation:

$$
E(f)=\frac{f}{f^{\prime}} E^{\prime}\left(f^{\prime}\right)
$$


The dielectric properties included in the FDTD method are specified at frequency $f$. As well as that, modeling of lowfrequency magnetic propagation in the human body converge in far less than a complete cycle, due to the small size of the modeled volume when compared to the wavelength [13]. Taking into account this aspect can significantly reduce the computing time. It has been demonstrated that such approximation gives correct results for ratios of up to 1:200000.

Table 1. Properties of the brain layers for the three frequencies considered in the FDTD simulations (50, 75 and $100 \mathrm{~Hz})$.

\begin{tabular}{lccccc}
\hline & & Skin and skull & Cerebrospinal fluid & Gray matter & White matter \\
\hline \hline \multirow{2}{*}{$\mathbf{5 0 ~ H z}$} & $\varepsilon[\mathrm{F} / \mathrm{m}]$ & $6.976 \cdot 10^{-6}$ & $9.638 \cdot 10^{-10}$ & $1.071 \cdot 10^{-4}$ & $4.677 \cdot 10^{-5}$ \\
& $\sigma[\mathrm{S} / \mathrm{m}]$ & $-2.565 \cdot 10^{-4}$ & $-\mathrm{i} \cdot 6.358 \cdot 10^{-3}$ & $-\mathrm{i} \cdot 2.392 \cdot 10^{-4}$ & $-\mathrm{i} \cdot 1.694 \cdot 10^{-4}$ \\
& $\mu_{r}$ & $8.070 \cdot 10^{-2}$ & 2 & $7.526 \cdot 10^{-2}$ & $5.327 \cdot 10^{-2}$ \\
& $\rho[\Omega / \mathrm{m}]$ & 1 & 1 & 1 & 1 \\
\hline \multirow{2}{*}{$\mathbf{7 5 ~ H z}$} & $\varepsilon[\mathrm{F} / \mathrm{m}]$ & $3.260 \cdot 10^{-6}$ & $9.638 \cdot 10^{-10}$ & $5.659 \cdot 10^{-5}$ & $2.402 \cdot 10^{-5}$ \\
& $\sigma[\mathrm{S} / \mathrm{m}]$ & $-\mathrm{i} \cdot 1.715 \cdot 10^{-4}$ & $-\mathrm{i} \cdot 4.238 \cdot 10^{-3}$ & $-\mathrm{i} \cdot 1.797 \cdot 10^{-4}$ & $-\mathrm{i} \cdot 1.199 \cdot 10^{-4}$ \\
& $\mu_{r}$ & $1.094 \cdot 10^{-2}$ & 2 & $8.482 \cdot 10^{-2}$ & $5.660 \cdot 10^{-2}$ \\
& $\rho[\Omega / \mathrm{m}]$ & 1 & 1 & 1 & 1 \\
\multirow{2}{*}{$\mathbf{1 0 0 ~ H z}$} & $\varepsilon[\mathrm{F} / \mathrm{m}]$ & $1.919 \cdot 10^{-6}$ & $9.638 \cdot 10^{-10}$ & $3.454 \cdot 10^{-5}$ & $1.475 \cdot 10^{-5}$ \\
& $\sigma[\mathrm{S} / \mathrm{m}]$ & $8.103 \cdot 10^{-2}$ & $-\mathrm{i} \cdot 3.179 \cdot 10^{-3}$ & $-\mathrm{i} \cdot 1.415 \cdot 10^{-4}$ & $-\mathrm{i} \cdot 9.233 \cdot 10^{-5}$ \\
& $\mu_{r}$ & 1 & 2 & $8.902 \cdot 10^{-2}$ & $5.809 \cdot 10^{-2}$ \\
& $\rho[\Omega / \mathrm{m}]$ & 12.34 & 1 & 1 & 1 \\
& & 1.35 & 0.5 & 11.23 & 17.21 \\
\hline
\end{tabular}

We have developed our own FDTD code taking into account these approximations. The method uses a three-dimensional realistic head mesh publicly available (namely Colin27 adult brain atlas FEM mesh Version 2) [14]. The total simulation volume is $212 \times 240 \times 208 \mathrm{~mm}$. We consider stimulation by a unitary magnetic dipole at three different frequencies, namely 50, 75 and $100 \mathrm{~Hz}$. The source position is $(\mathrm{x}, \mathrm{y}, \mathrm{z})=(75.76,66.99,168.22) \mathrm{mm}$. Dielectric properties of the skull, cerebrospinal fluid, grey matter and white matter have been taken from the available literature [15], and are shown in Table 1. According to the referenced approximations, modeling is performed at $1 \mathrm{MHz}$ and later scaled to each frequency of interest. The maximum simulation time is $50 \mathrm{~ns}$ in all cases. The rest of parameters involved in the FDTD calculation are included in Table 2.

Table 2. Space and time discretization parameters for the FDTD method at 50, 75 and $100 \mathrm{~Hz}$.

\begin{tabular}{cccc}
\hline & $\mathbf{5 0 ~ H z}$ & $\mathbf{7 5 ~ H z}$ & $\mathbf{1 0 0 ~} \mathbf{~ H z}$ \\
\hline \hline$\Delta_{i j k}[\mathrm{~mm}]$ & 4.311 & 5.929 & 7.590 \\
$\left(N_{x}, N_{y}, N_{z}\right)$ & $(51,57,50)$ & $(37,42,37)$ & $(29,33,29)$ \\
Total number of cells & 145350 & 57498 & 27753 \\
$\Delta_{t}[\mathrm{ps}]$ & 8.3 & 11.4 & 14.6 \\
Number of time steps & 6027 & 4382 & 3423 \\
\hline
\end{tabular}

In Fig. 3 we show the electric field spatial distribution in the brain cortex (surface of the grey matter) calculated by the FDTD method for the three simulated frequencies. Results are given in relative decibels with respect to the maximum value in the head surface, and are in agreement with the typical values [13]. The results enable to analyze the radiation distribution with a high degree of detail and specificity. Note that the scale has been fixed the same for the three cases. The radiation distribution pattern is similar in the three modeled situations. It can be observed that the lack of directivity of the magnetic dipole results in the magnetic irradiation of very large brain areas, which can be avoided by using magnetic coils with specific designs [3]. 


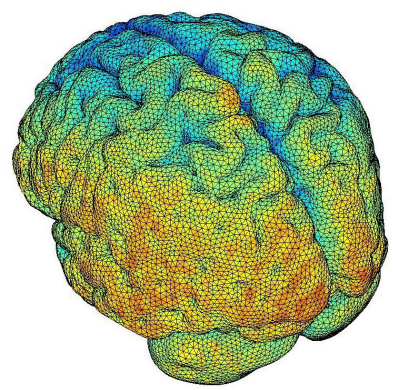

a)

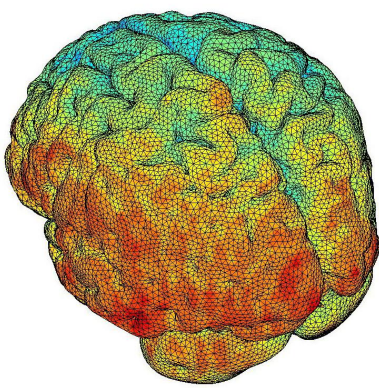

b)

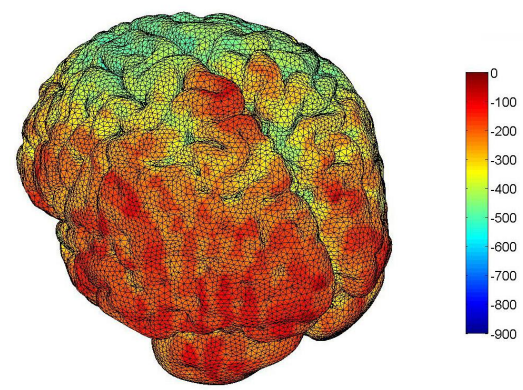

c)

Fig. 3. Electric field distribution in cerebral cortex for irradiation with a unitary magnetic dipole at (a) 50 , (b) 75 and (c) 100 Hz. Values are given in relative $\mathrm{dB}$ with respect to the maximum value in the outer layer of the head.

In order to better appreciate the characteristics of the electromagnetic field distribution in the brain, the coronal plane at $\mathrm{y}=70 \mathrm{~mm}$ are shown in Figure 4. The different brain layers have also been included, where gray corresponds to the skin and skull, purple is associated with the cerebrospinal fluid, green represents gray matter, and blue is white matter. It is interesting to highlight that the head is embedded in magnetic radiation, due to the fact that the wavelength is extremely high in comparison to the modeled volume at the source frequency used in the simulations, apart from the nondirectional coil used as a source.
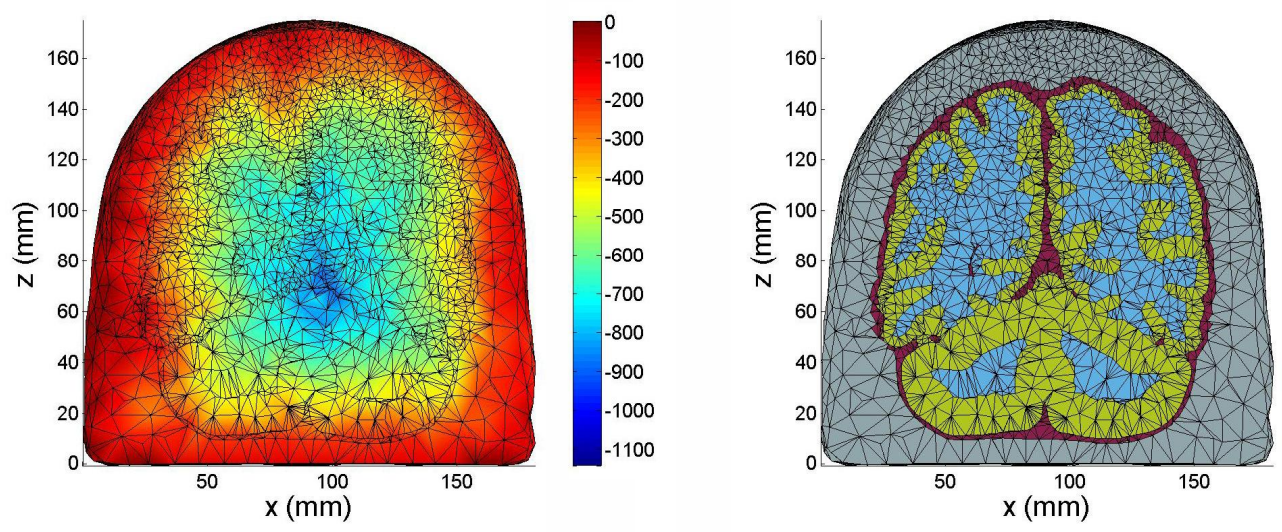

Fig. 4. Electric field distribution in cerebral cortex for irradiation with a unitary magnetic dipole at $75 \mathrm{~Hz}$ for the coronal plane at $\mathrm{y}=70 \mathrm{~mm}$. Values are given in relative $\mathrm{dB}$ with respect to the maximum value in the outer layer of the head.

Regarding the Monte Carlo method for modeling light propagation, in this work we use the open-source Mesh-based Monte Carlo method recently developed by Fang [14]. We consider two different types of sources: a single collimated source with a wavelength of $632.8 \mathrm{~nm}$ (corresponding to a HeNe laser) located at $(\mathrm{x}, \mathrm{y}, \mathrm{z})=(0,155,115) \mathrm{mm}$, and five collimated sources at the same wavelength located at a height of $z=115 \mathrm{~mm}$ and equally spaced $15 \mathrm{~mm}$ between each other from $\mathrm{y}=125 \mathrm{~mm}$ to $\mathrm{y}=185 \mathrm{~mm}$. Optical properties of the different head layers are specified for the source wavelength [15] and included in Table 3.

Table 3. Optical properties of the human skin layers used in the Monte Carlo method.

\begin{tabular}{ccccc}
\hline Brain layer & Refractive index & $\mu_{a}\left[\mathbf{m m}^{-1}\right]$ & $\mu_{s}\left[\mathbf{m m}^{-1}\right]$ & Scattering anisotropy \\
\hline \hline Skin and skull & 1.37 & 0.019 & 7.8 & 0.89 \\
Cerebrospinal fluid & 1.37 & 0.004 & 0.009 & 0.89 \\
Gray matter & 1.37 & 0.02 & 9.0 & 0.89 \\
White matter & 1.37 & 0.08 & 40.9 & 0.84 \\
\hline
\end{tabular}



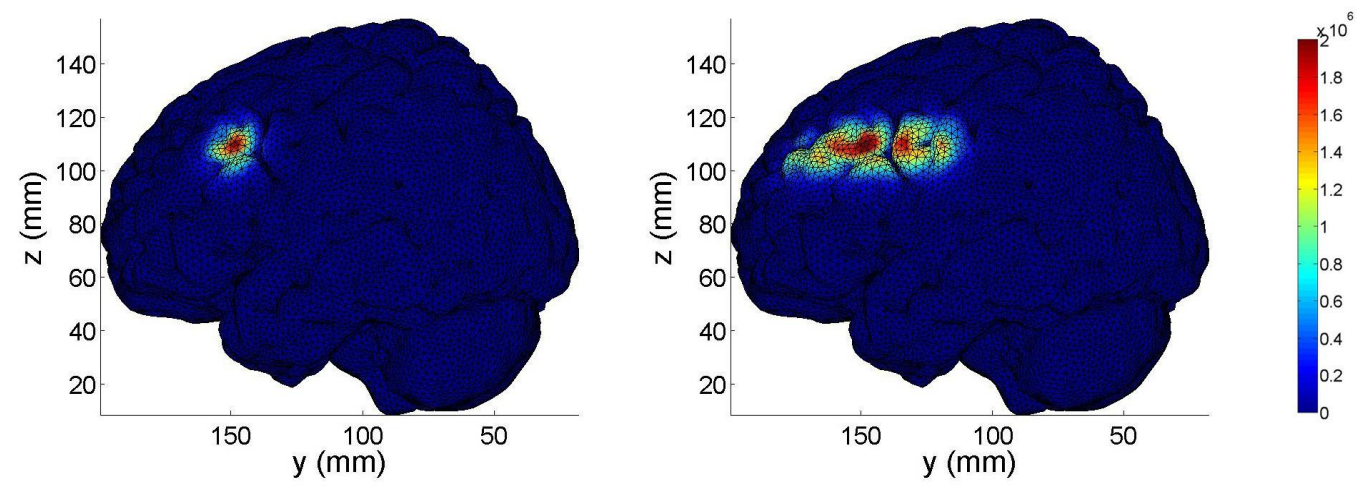

Fig. 5. Photons distribution in the cerebral cortex for illumination with a single optical source (left) and an array of five optical sources with an inter-source spacing of $15 \mathrm{~mm}$. The source corresponds to a HeNe laser. Values are given in number of photons per volumetric element.

The photons distribution calculated by the Mesh-based Monte Carlo method in both cases has been depicted in Figure 5, which represents the number of photons in each volumetric element. It can be appreciated that optical illumination shows a high degree of spatial specificity, which enables to confine photons in a very narrow region of the head. As well as that, it enables to compare the characteristics of single and multiple sources for optically stimulating human brain. The aim of using multiple light sources is to achieve the stimulation of a broader brain region. In this case, it can be observed that the region reached by optical radiation in the cortex region is significantly larger for multiple optical sources, achieving a reasonably uniform illumination over the region of interest.

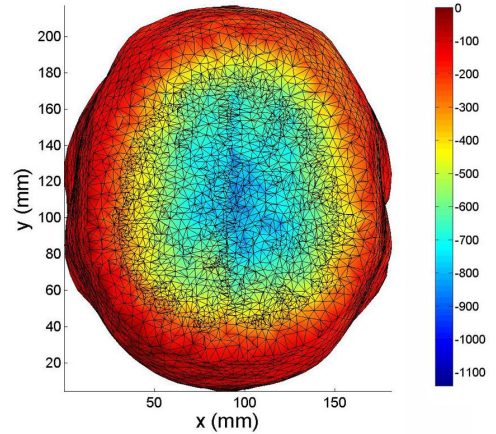

a)

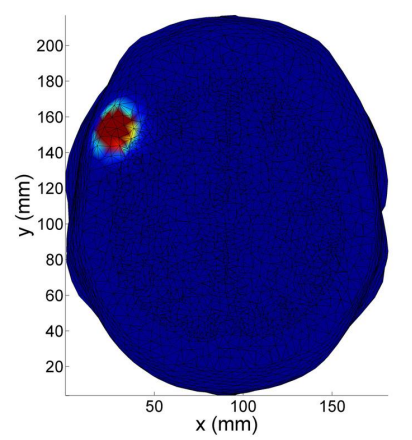

c)

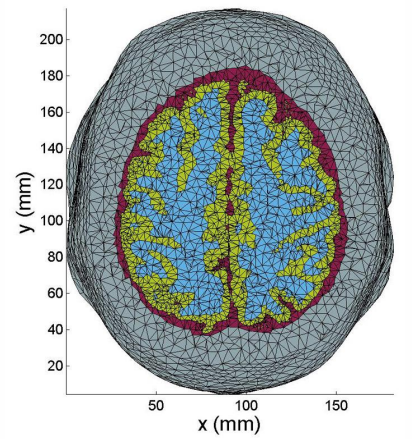

b)

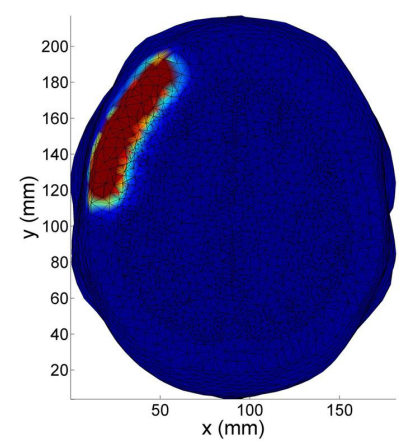

d)

Fig. 6. Spatial distribution of the electric field (a), brain layers (b), photons distribution with a single optical source (c) and an array of five optical sources (d) for the transversal plane at $z=115 \mathrm{~mm}$. The scale of (c) and (d) is the same. 
Finally, FDTD and Monte Carlo calculations of magnetic and optical propagation are compared in Fig. 6 in order to complement the results presented above. Specifically, the spatial distribution of the electric field at $75 \mathrm{~Hz}$ (a) and the different brain layers (b) have been depicted for the transversal plane $\mathrm{z}=115 \mathrm{~mm}$. Additionally, photons distribution for a single (c) and multiple (d) optical sources at the same transversal plane have been included. Results are given in the same units as above. This Figure enables to clearly appreciate the differences in electromagnetic propagation when using low-frequency magnetic sources and collimated optical sources. In the first case, electromagnetic radiation affects a very large region of cerebral tissues, and progressively decreases from the cortex to the white matter. However, when illuminating with optical sources, energy deposition is confined to a specific narrow volume within the head, reaching a small region of the cortex and rapidly decreasing inside the grey matter. The versatility of using multiple sources for optical brain stimulation can also be appreciated.

\section{CONCLUSIONS}

In this work, the FDTD method and the Monte Carlo method have been applied to a three-dimensional realistic adult head mesh for respectively modeling magnetic and optical propagation in human brain. The results show that these methods enable to predict the radiation distribution in different regions of the brain for both types of irradiation. The characteristics of the presented results confirm the potential of both numeric methods. The high resolution of the results enables to accurately predict the radiation distribution in the different layers of the brain, which is very interesting for developing robust predictive models for magnetic and optical stimulation techniques like TBS and INS. Regarding optical radiation, it is also interesting to highlight that different illumination patterns can be achieved by irradiating with an array of optical sources, which can be very useful in practical situations. Specifically, such characteristic entails a high potential for stimulating specific functional regions in the cerebral cortex.

\section{ACKNOWLEDGEMENTS}

This work has been partially carried out with the support of San Cándido Foundation.

\section{REFERENCES}

[1] European Technology Platform Photonics 21, "Photonics 21 Strategic Research Agenda" (2010).

[2] Wells, J., Kao, C., Mariappan, K., Albea, J., Jansen, E. D., Konrad, P., Mahadevan-Jansen, A., "Optical stimulation of neural tissue in vivo," Optics Letters 30, 504-506 (2005).

[3] Groppa, S. et al. "A practical guide to diagnostic transcranial magnetic stimulation: Report of an IFCN committee", Clinical Neurophysiology 123, 858-882 (2012).

[4] Wu, A. D., Fregni, F., Simon, D. K., Deblieck, C., Pascual-Leone, A., "Noninvasive Brain Stimulation for Parkinson's Disease and Dystonia", Neurotherapeutics: The Journal of the American Society for Experimental NeuroTherapeutics 5, 345-361 (2008).

[5] Bentwich, J., Dobronevsky, E., Aichenbaum, S., Shorer, R., Peretz, R., Khaigrekht, M., Marton, R. G., Rabey, J. M., "Beneficial effect of repetitive transcranial magnetic stimulation combined with cognitive training for the treatment of Alzheimer's disease: a proof of concept study", J Neural Transm 118, 463-471 (2011).

[6] Richter, C.-P., Izzo Matic, A., Wells, J. D., Jansen, E. D., Walsh Jr., J. T., "Neural stimulation with optical radiation," Laser \& Photonics Reviews 5, 68-80 (2011).

[7] Taflove, A., Hagness, S., [Computational Electrodynamics: The Finite Difference Time Domain Method], Artech House (2000).

[8] Romanov, O. G., Tolstik, A. L., Ortega-Quijano, N., Fanjul-Vélez, F., Arce-Diego, J. L., "Finite-difference timedomain simulation of light propagation in 2D scattering media," Nonlinear Phenomena in Complex Systems 12, 267-274 (2009).

[9] Pontalti, R., Nadobny, J., Wust, P., Vaccari, A., Sullivan, D., "Investigation of static and quasi-static fields inherent to the pulsed FDTD method," IEEE Trans. Microwave Theory Techniques 50, 2022-2025 (2002).

[10] Wang, L. V., Wu, W., [Biomedical Optics. Principles and Imaging], Wiley (2007).

[11] Ortega-Quijano, N., Fanjul-Vélez, F., de Cos-Pérez, J., Arce-Diego, J. L., "Analysis of the depolarizing properties of normal and adenomatous polyps in colon mucosa for the early diagnosis of precancerous lesions," Optics Communications 284, 4852-4856 (2011). 
[12] Wang, L. H., Jacques, S. L., Zheng, L. Q., "Monte Carlo modeling of light transport in multi-layered tissues," Computer Methods and Programs in Biomedicine 47, 131-146 (1995).

[13] Furse, C. M., "Application of the Finite-Difference Time-Domain method to bioelectromagnetic simulations," Applied Computational Electromagnetics Society Newsletter (1997).

[14] Fang, Q., "Mesh-based Monte Carlo method using fast ray-tracing in Plucker coordinates," Biomed. Opt. Express 1, $165-175$ (2010).

[15] Gabriel, S., Lau, R. W., Gabriel, C., "The dielectric properties of biological tissues: II. Measurements in the frequency range $10 \mathrm{~Hz}$ to $20 \mathrm{GHz}$, Phys. Med. Biol. 41, 2251-2269 (1996). 